\title{
A Class of Thickness-Minimal Graphs
}

\author{
Arthur M. Hobbs and J. W. Grossman \\ Institute for Applied Technology, National Bureau of Standards, Washington, D.C. 20234
}

(April 8, 1968)

\begin{abstract}
The thickness of a graph $G$ is the minimum number of planar subgraphs whose union is $G$. A $t$-minimal graph is a graph of thickness $t$ which contains no proper subgraph of thickness $t$. In this paper, we show that the complete bipartite graph on two sets each containing $4 t-5$ vertices is $t$-minimal for all $t \geqslant 2$. We also show that if the complete graph on 16 vertices has thickness 4 , it is 4-minimal.

Key Words: Bipartite graphs, graph theory, mathematics, planar graphs, thickness of graphs, $t$-minimal graphs.
\end{abstract}

A graph is a finite set of points, called vertices, together with a finite set of line segments, called edges, such that each end point of an edge is a vertex and two edges meet only at an end point of each. The end points of an edge are said to be joined by the edge. A subgraph $H$ of a graph $G$ is a subset of the edges and vertices of $G$ such that the end points of every edge in $H$ are also in $H$.

A graph is planar if it can be drawn in the plane in such a way that no two distinct points of the graph are mapped to the same point of the plane. The thickness $t(G)$ of a graph $G$ is defined [7] ${ }^{1}$ as the minimum number of planar subgraphs whose union is G. A thickness-minimal, or $t$-minimal, graph is a graph of thickness $t$ which contains no proper subgraph of thickness $t$.

A graph is complete if every pair of vertices is joined by an edge; a complete graph with $n$ vertices is denoted by $K_{n}$. A graph $G$ is bipartite if there is a separation of the vertices of $G$ into two disjoint sets $A$ and $B$ such that every edge of $G$ joins a vertex in $A$ with a vertex in $B$. A bipartite graph is complete if every vertex in $A$ is joined to every vertex in $B$; such a graph is denoted $K_{m, n}$, where $m$ is the number of vertices in the set $A$ and $n$ is the number of vertices in the set $B$.

In 1930 Kuratowski [6] proved that the only 2-minimal graphs are the homeomorphs of $K_{5}$ and $K_{3,3}$. In $1963 \mathrm{~W}$. T. Tutte [7] showed that there exists an infinite set of nonhomeomorphic $t$-minimal graphs for each $t \geqslant 3$. However, the only known $t$-minimal graph for $t \geqslant 3$ has been $K_{9}$, which is 3 -minimal $[1,7,8]$.

In this paper we show that $K_{4 t-5,4 t-5}$ is $t$-minimal for all $t \geqslant 2$. We also give an embedding, in three planes, of $K_{16}$ less one edge; hence if (as F. Harary suspects [5]) $K_{16}$ has thickness 4 , then it is 4-minimal.

LEMMA 1: $\mathrm{t}\left(\mathrm{K}_{4 \mathrm{t}-5,4 \mathrm{t}-5)}\right) \mathrm{t}$ for all $\mathrm{t} \geqslant 2$.

This lemma is a consequence of a more general theorem proved by Beineke, Harary, and Moon [4]. For completeness we include a simple proof of our lemma:

Proof: Let $H_{1}, \ldots, H_{t-1}$ be $t-1$ planar subgraphs of $K_{4 t-5,4 t-5}$, each $H_{i}$ containing all $8 t-10$ vertices in $K_{4 t-5,4 t-5}$. Since the smallest circuit in a bipartite graph contains at least four edges, the number of faces $F_{i}$ in each $H_{i}$ is related to the number of edges $E_{i}$ in $H_{i}$ by

or

$$
4 F_{i} \leqslant 2 E_{i}
$$

$$
F_{i} \leqslant 1 / 2 E_{i} .
$$

${ }^{1}$ Figures in brackets indicate the literature references at the end of this paper. 
By Euler's formula for the plane and inequality (1),

$$
8 t-10-E_{i}+1 / 2 E_{i} \geqslant 2,
$$

or

$$
E_{i} \leqslant 16 t-24
$$

Summing over all $t-1$ planar subgraphs $H_{i}$, at most

$$
E^{\prime}=(t-1)(16 t-24)=16 t^{2}-40 t+24
$$

edges of $K_{4 t-5,4 t-5}$ can be contained in $t^{-1}$ planar subgraphs. But $K_{4 t-5,4 t-5}$ contains

$$
E=(4 t-5)^{2}=16 t^{2}-40 t+25
$$

edges, and $E>E^{\prime}$.

Thus, if we show that $K_{4 t-5,4 t-5}$ less one edge can be embedded in $t-1$ planes, we will have shown that it has thickness exactly $t$ and is in fact $t$-minimal. We shall represent $K_{n, n}$ as the set of vertices

$$
\left\{1,2, . . ., n, A_{1}, A_{2}, \ldots ., A_{n}\right\}
$$

together with all edges joining a numbered vertex to a lettered vertex. By symmetry, the choice of the only edge to be placed in the $t$ 'th plane is arbitrary; we shall always use edge $\left(1, A_{1}\right)$. Since the construction of our main theorem is invalid for $K_{7,7}$, we need

LEMMA 2: $\mathrm{K}_{7,7}$ is 3-minimal.
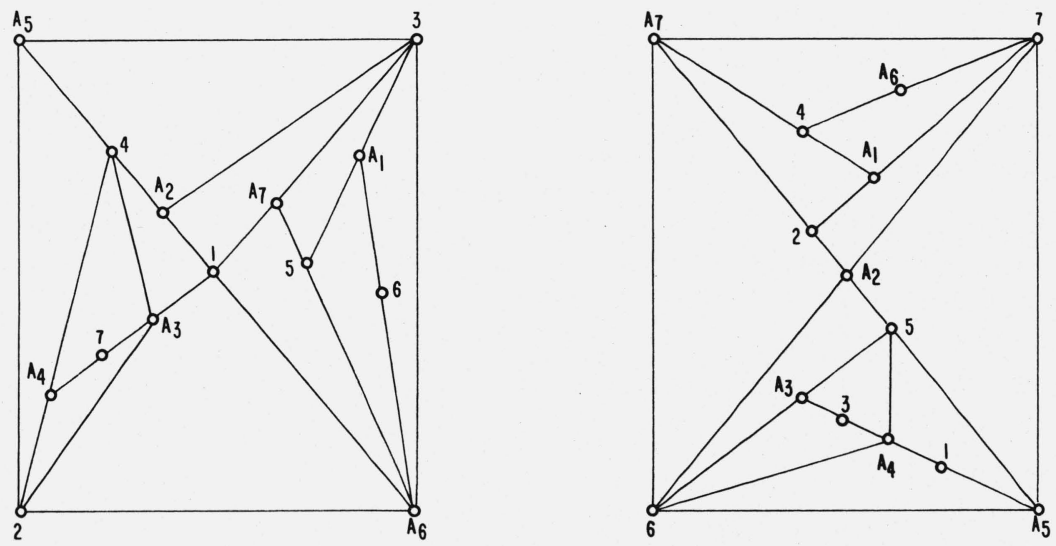

FIGURE 1. $\mathrm{K}_{7,7}$ less one edge is biplanar. (Edge $\left(1, \mathrm{~A}_{1}\right)$ is missing).

Proof: By Lemma $1, K_{7}, 7$ has thickness at least 3 . But figure 1 shows that $K_{7,7}$ less the edge $\left(1, A_{1}\right)$ can be embedded in two planes.

QED

The following theorem is proven by construction; we have made this construction subject to the extra condition that the lettered vertices are placed on a circle in the same order in each of the planes and no edge of the graph may cross the circle in any plane.

A numbered vertex in Plane $N$ is said to be simply joined in that plane if it is joined by edges to exactly two lettered vertices which are adjacent on the circle. Clearly any number of numbered vertices may be simply joined to lettered vertices in any plane without affecting the remaining construction. 
THEOREM: $\mathrm{K}_{4 \mathrm{t}-5,4 \mathrm{t}-5}$ is $\mathrm{t}$-minimal for all $\mathrm{t} \geqslant 2$.

Proof: By the well known result for $K_{3,3}$ and Lemmas 1 and 2 , we need only embed $K_{4 t-5,4 t-5}$ less edge $\left(1, A_{1}\right)$ in $t-1$ planes for each value of $t \geqslant 4$. To begin the construction, illustrated in figure 2 for $K_{15,15}$, place the lettered vertices on a circle in each of the $t-1$ planes, using the same order around the circle in each of the planes (we have used $A_{1}, A_{2}, \ldots$., $A_{4 t-5}$ in clockwise order).

1. Place vertex 1 outside the circle in Plane 1 and join it to the $2 t-2$ consecutive lettered vertices $A_{2}, A_{3}, \ldots, A_{2 t-1}$. Since edge $\left(1, A_{1}\right)$ is excluded from the $t-1$ planes, we complete vertex 1 by simple joins in Planes $2,3, \ldots, t-1$, joining it to vertices $A_{2 t}$ and $A_{2 t+1}$ in Plane 2, to $A_{2 t+2}$ and $A_{2 t+3}$ in Plane $3, \ldots$, and to $A_{4 t-6}$ and $A_{4 t-5}$ in Plane $t-1$.

Call the region in Plane 1 outside the circle bounded by the portion of the circle having lettered vertices not joined to vertex 1 and by the two edges $\left(1, A_{2}\right)$ and $\left(1, A_{2 t-1}\right)$ the large outside region of Plane 1.

2. Place vertex 2 inside the circle in Plane $p, p=2,3, \ldots, t-1$, and join it to vertices $A_{2 p-3}$ and $A_{2 p+2 t-6}$ in that plane.

Note that the connections made in step 2 split the interior of the circle in each of Planes 2 through $t-1$ into two parts. One is bounded by the two edges joined to vertex 2 and a portion of circle containing $2 t-2$ lettered vertices; let us call this the small inside region of Plane $p$. The other part of the interior is bounded by the two edges joined to vertex 2 and the remaining part of the circle, containing $2 t-1$ lettered vertices; we call this region the large inside region.

3. Place vertex 2 inside the circle in Plane 1 and join it to the lettered vertices to which it was not connected in step 2 , namely to the lettered vertices with subscript $2,4,6, \ldots ., 2 t-4$, $2 t-3,2 t-1,2 t+1, \ldots ., 4 t-7,4 t-6$, and $4 t-5$.

Step 3 completes the connection of vertex 2. Define a gap to be a region in Plane 1 bounded by a segment of the circle having three consecutive lettered vertices on it, the middle one not being connected to vertex 2 , and by the edges joining vertex 2 to the other two lettered vertices

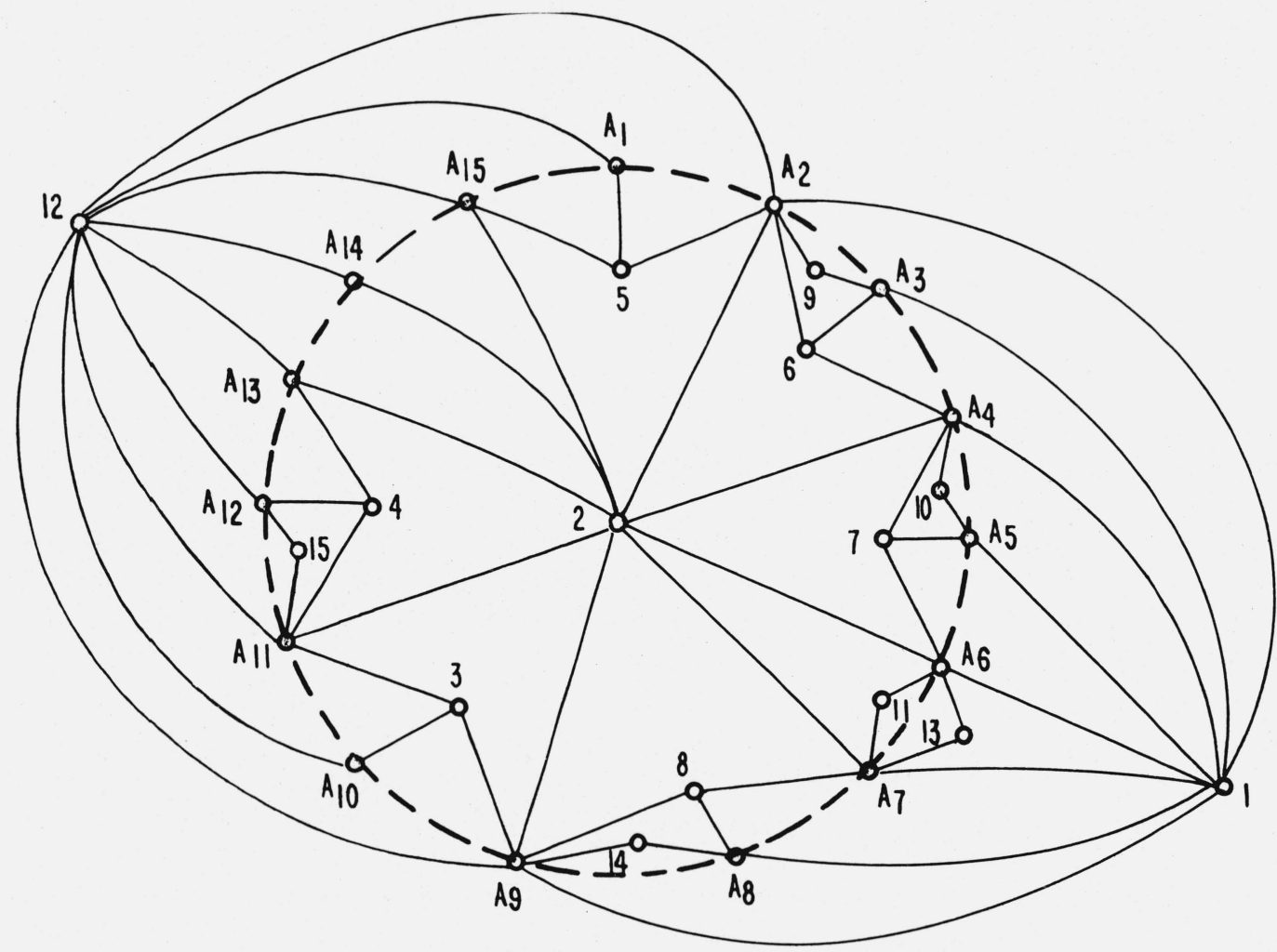

Figure 2. Plane 1 of $\mathrm{K}_{15,15}$. 
on the segment. There are $2(t-2)=2 t-4$ gaps in Plane 1 . A vertex which is joined to $2 t-2$ lettered vertices in one plane cannot be completed by simple joins in the remaining $t-2$ planes, since using only simple joins would leave it connected to only $2 t-2+2(t-2)=4 t-6$ lettered vertices, one short of the required number. We shall use the gaps in Plane 1 to make up the deficit during the next few steps.

4. In Plane $p, p=2,3, \ldots, t-2$, place vertex $p+1$ inside the small inside region and join it to the $2 t-2$ lettered vertices having subscripts $2 p-3,2 p-2, \ldots ., 2 p+2 t-6$ on the boundary of that region.

Note that vertex 2 is joined to vertices $A_{2 p-3}$ and $A_{2 p+2 t-6}$ in Plane $p$, so that the lettered vertices named really are on the boundary of the small region.

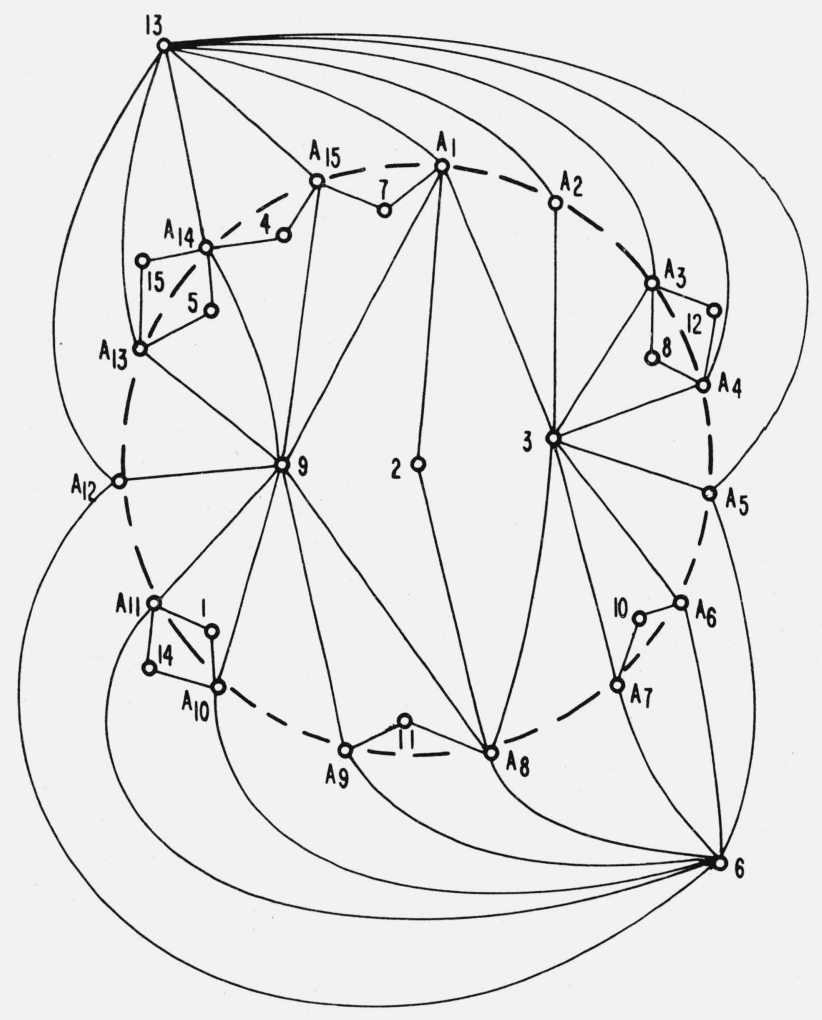

Figure 2. Plane 2 of $\mathrm{K}_{15,15}$.

5. For $p=2,3, \ldots, t-2$, place vertex $p+1$ inside the gap in Plane 1 which has lettered vertices $A_{2 p+2 t-5}, A_{2 p+2 t-4}$, and $A_{2 p+2 t-3}$ on its boundary. Join vertex $p+1$ to those three lettered vertices.

The gap required by step 5 exists because in step 2 vertex 2 was joined to the lettered vertex having subscript $2(p+1)+2 t-6=2 p+2 t-4$ in Plane $p+1$ for each $p=2,3, \ldots ., t-2$.

6 . For $p=2,3, \ldots, t-2$, join vertex $p+1$ to the remaining $2 t-6$ lettered vertices by using simple joins in the planes other than 1 and $p$.

7. In Plane $t-1$, place vertex $t$ inside the small region, which has the lettered vertices with subscripts $2 t-5,2 t-4, \ldots ., 4 t-8$ on its boundary, and join vertex $t$ to those lettered vertices.

8. Place vertex $t$ in the gap in Plane 1 having lettered vertices with subscripts $4 t-5,1$, and 2 on its boundary (this gap exists since vertex 2 is joined to vertex $A_{1}$ in Plane 2) and join vertex $t$ to those lettered vertices.

9. In Plane 2, place vertex $t$ between vertices $A_{4 t-7}$ and $A_{4 t-6}$ and join it to those vertices, a simple join.

10. Using simple connections in Planes $3,4, \ldots, t-2$, join vertex $t$ to the remaining $2 t-8$ consecutive lettered vertices $A_{3}, A_{4}$. . . ., $A_{2 t-6}$. 


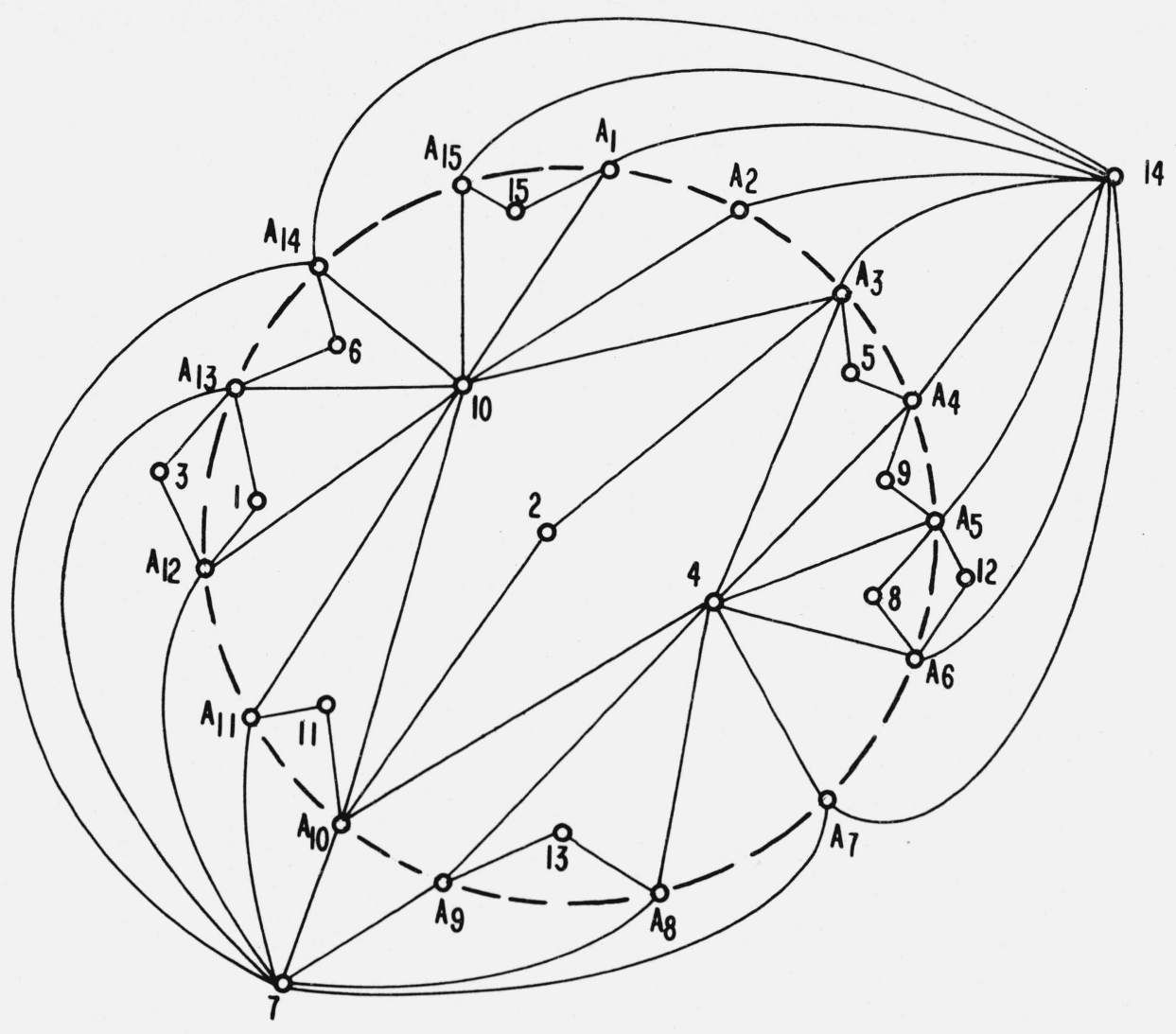

Figure 2. Plane 3 of $\mathrm{K}_{15,15}$.

Now we have used $t-2$ of the gaps in Plane 1, leaving us with $t-2$ more gaps, and we have not placed any edges or vertices outside the circle in any of Planes $2, \ldots, t-1$.

11. In Plane $p, p=2, \ldots, t-2$, place vertex $p+t-1$ outside the circle and join it to the $2 t-2$ consecutive lettered vertices having the subscripts $2 p+1,2 p+2, \ldots ., 2 p+2 t-2$.

Call the region bounded by the rest of the circle and the two edges $\left(p+t-1, A_{2 p+1}\right)$ and $\left(p+t-1, A_{2 p+2 t-2}\right)$ the large outside region of Plane $p$. In step 11 vertex $p+t-1$ is joined to the $2 t-2$ lettered vertices immediately following the three lettered vertices on the boundary of the still unused gap produced by the connection of vertex 2 to vertex $A_{2 p-1}$ in Plane $p+1$. Hence the following step:

12. In Plane 1 , place vertex $p+t-1, p=2, \ldots, t-2$, in the gap having vertices $A_{2 p-2}$, $A_{2 p-1}$, and $A_{2 p}$ on its boundary, and join vertex $p+t-1$ to those three lettered vertices. Use simple joins to complete the connections of vertex $p+t-1$ in the planes other than 1 and $p$.

13. In Plane $t-1$, place vertex $2 t-2$ outside the circle and join it to the $2 t-2$ consecutive lettered vertices having subscripts $2 t, 2 t+1, \ldots, 4 t-5,1$, and 2 . Place vertex $2 t-2$ in Plane 1 in the one remaining unused gap, having vertices $A_{2 t-3}, A_{2 t-2}$, and $A_{2 t-1}$ on its boundary, and join vertex $2 t-2$ to those lettered vertices. Complete vertex $2 t-2$ with simple joins in Planes 2 , $3, . ., t-2$.

Call the region bounded by the rest of the circle in Plane $t-1$ and by the two edges $(2 t-2$, $\left.A_{2 t}\right)$ and $\left(2 t-2, A_{2}\right)$ the large outside region of Plane $t-1$. Now each of the large inside regions and large outside regions in Planes 1 through $t-1$ has $2 t-1$ lettered vertices on its boundary. A numbered vertex placed in any one of these regions can be joined to $2 t-1$ consecutive lettered vertices there, leaving only $4 t-5-(2 t-1)=2 t-4=2(t-2)$ consecutive lettered vertices to be joined to it. These remaining connections can easily be completed by simple joins in the remaining planes. Specifically: 


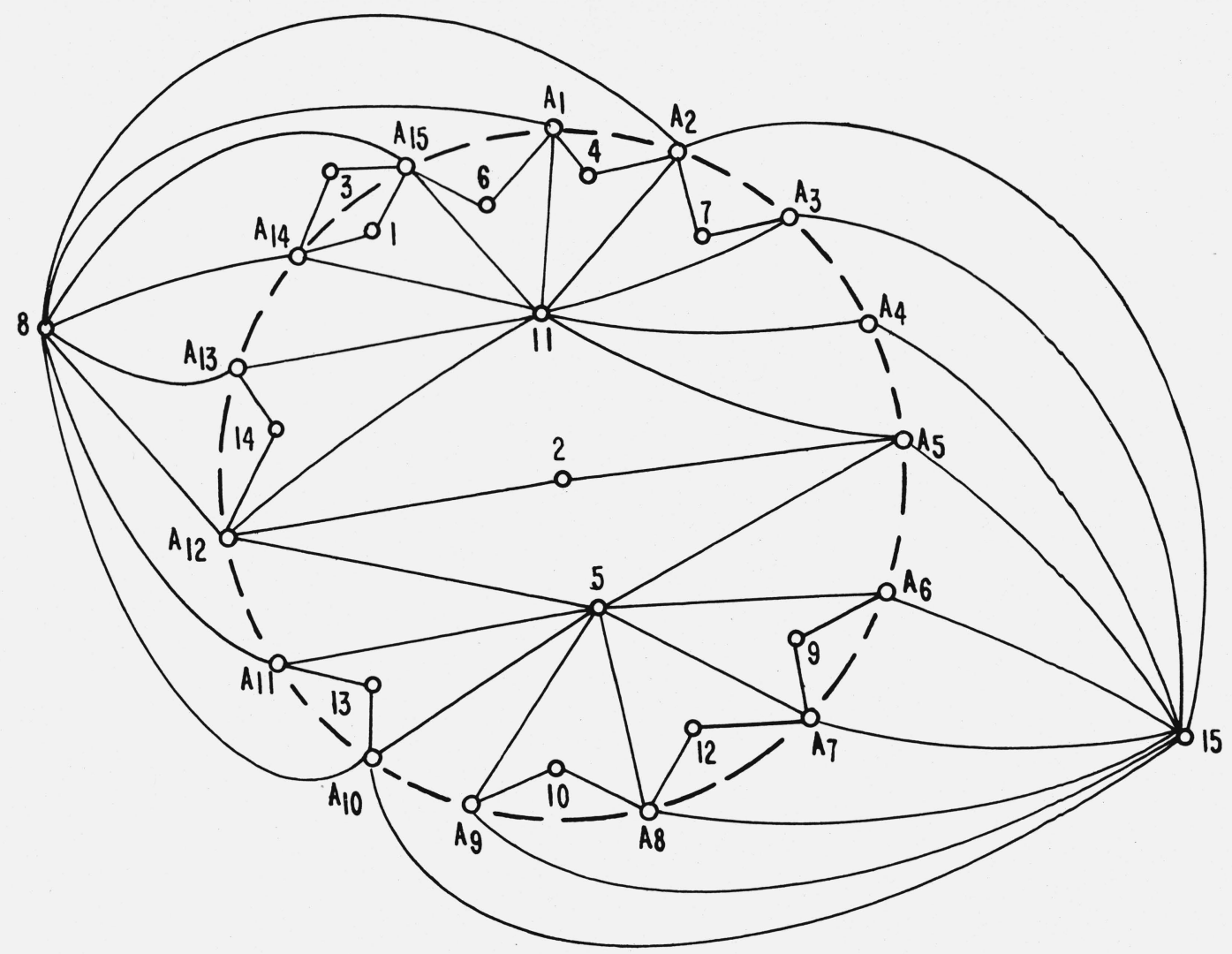

Figure 2. Plane 4 of $\mathrm{K}_{15,15}$.

14. In Plane $p, p=2,3, \ldots, t-1$, place vertex $p+2 t-3$ inside the large inside region and join the vertex to the lettered vertices with subscripts $2 p+2 t-6,2 p+2 t-5, \ldots ., 4 t-5,1$, $2, \ldots, 2 p-3$. Complete vertex $p+2 t-3$ in the remaining $t-2$ planes with simple joins.

15. In Plane $p, p=1,2, \ldots, t-1$, place vertex $p+3 t-4$ in the large outside region and join the vertex to the $2 t-1$ consecutive lettered vertices on the boundary of the region. Complete vertex $p+3 t-4$ in the remaining $t-2$ planes with simple joins.

This completes the construction.

It will be noticed that the connections of vertex 2 play a key role in carrying out the above construction. A gap is created in Plane 1 because of the connections of vertex 2 in some Plane $p+1$, and this gap is used by a vertex whose other nonsimple joins are in Plane $p$ (or in Plane $t-1$ for the case of $p=1$ ). It is this interdependence of the connections in three planes at once that prevents the use of the above construction for $K_{7,7}$, for which $t-1=2$, and thus requires Lemma 2.

Another possible class of $t$-minimal graphs is $\left\{K_{\varphi(t)}\right\}$ for some appropriate function $\varphi(t)$. Since $K_{5}$ is 2 -minimal and $K_{9}$ is 3 -minimal, we know the value of $\varphi(t)$ for $t=2$ and 3 . The thickness of $K_{16}$ is known to be either 3 or 4 . Figure 3 shows that $K_{16}$ less edge $(1,16)$ can be embedded in three planes; therefore, if $K_{16}$ has thickness 4 , it is 4-minimal. See Beineke and Harary [2 and 3] for further results on $t\left(K_{n}\right)$.

The authors thank Jack Edmonds for his many invaluable suggestions during the preparation of this paper. 


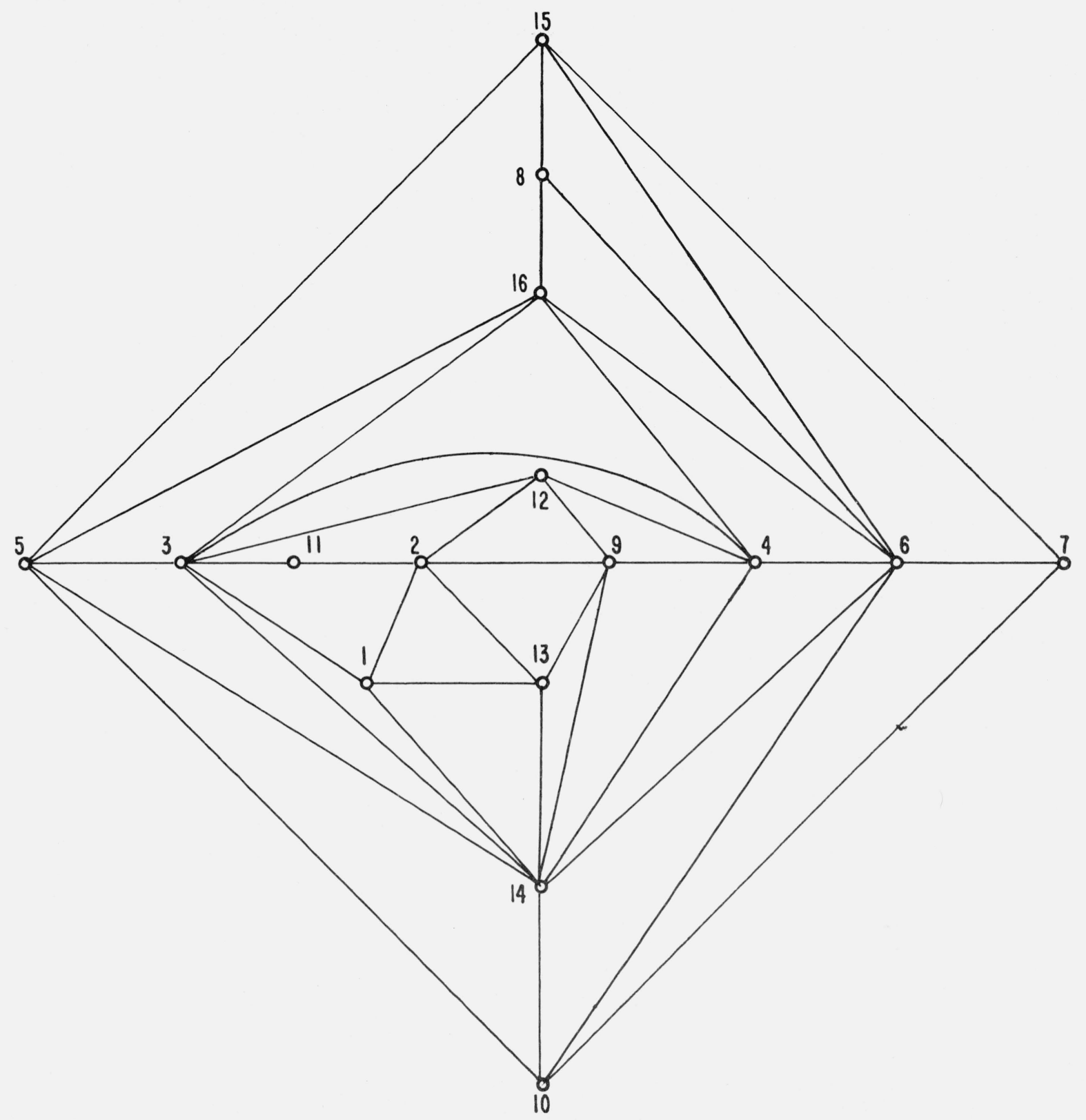

Figure 3. Plane 1 of $\mathrm{K}_{16}$ less edge $(1,16)$.

151 


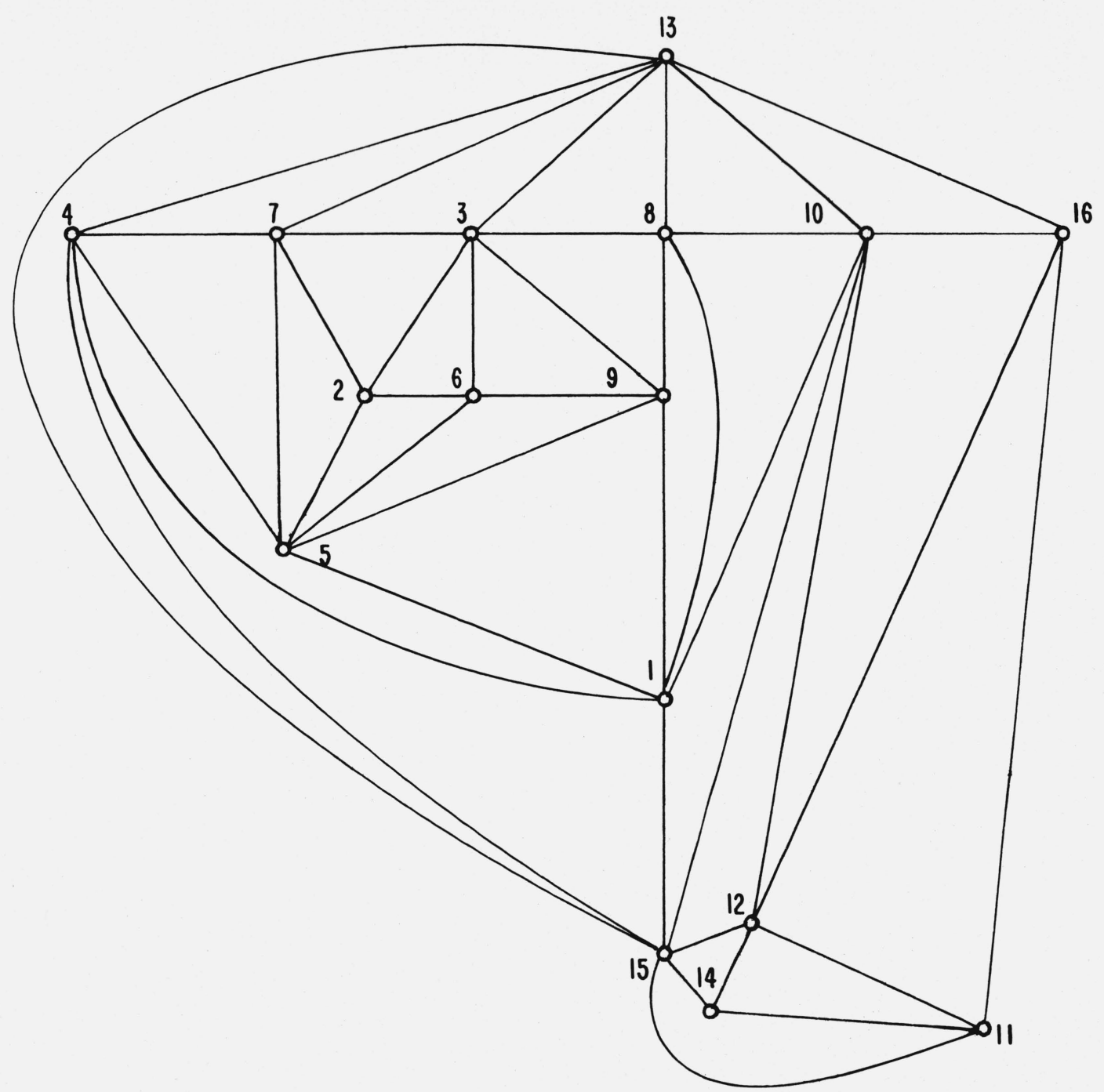

Figure 3. Plane 2 of $\mathrm{K}_{16}$ less edge $(1,16)$. 


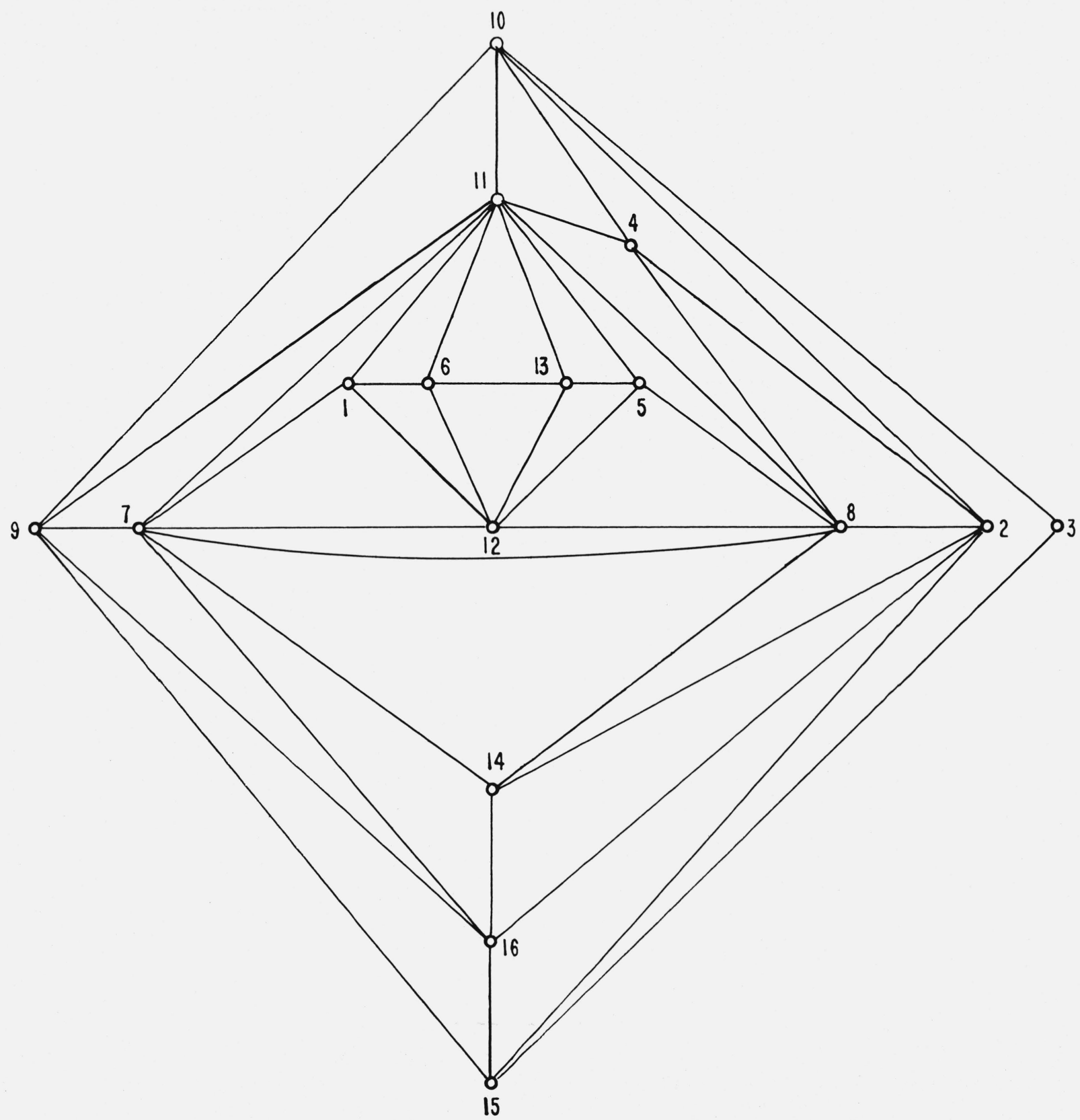

Figure 3. Plane 3 of $\mathrm{K}_{16}$ less edge $(1,16)$.

\section{References}

[1] J. Battle, F. Harary, and Y. Kodama, Every planar graph with nine points has a non-planar complement, Bull. Amer. Math. Soc. 68, 569-571 (1962).

[2] L. W. Beineke, and F. Harary, Inequalities involving the genus of a graph and its thickness, Proc. Glasgow Math. Assoc. 7, 19-21 (1965)

[3] L. W. Beineke and F. Harary, The thickness of the complete graph, Canad. J. Math. 1 7, 850-859 (1965).

[4] L. W. Beineke, F. Harary, and J. W. Moon, On the thickness of the complete bipartite graph, Proc. Camb. Phil. Soc. 60, $1-5$ (1964).

[5] F. Harary, Recent results in topological graph theory, Acta Math. Acad. Sci. Hungar. 15, 405-413 (1964).

[6] C. Kuratowski, Sur le probleme des courbes gauches en topologie, Fund. Math. 15, 271-283 (1930).

[7] W. T. Tutte, The thickness of a graph, Nederl. Akad. Wetensch. Proc. Ser. A 66; Indag. Math. 25, 567-577 (1963).

[8] W. T. Tutte, The non-biplanar character of the complete 9-graph, Canad. Math. Bull. 6, 319-330 (1963).

(Paper 72B2-268) 